\title{
ANALYSIS OF HEAT AND MASS TRANSPORT PROCESSES NEAR AN EMPLACED \\ NUCLEAR WASTE CANISTER
}

Final Report

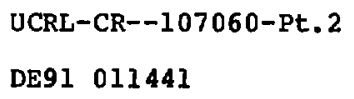

May 22, 1990

by

Carl Keller

SCIENCE AND ENGINEERING ASSOCIATES, INC. 612 Old Santa Fe Trail

Santa Fe, NM 87501

for

Dale Wilder

LAWRENCE LIVERMORE NATIONAL LABORATORY YUCCA MOUNTAIN PROJECT

LLNL Subcontract No. B055747

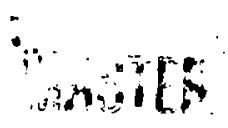




\section{BACKGROUND}

Science and Engineering Associates, Inc. (SEA) has assisted the Lawrence Liverwore National Laboratory in a review of the models and experiment plans for evaluation of the spent fuel canister environment in a nuclear repository. Our special emphasis was on the relevance of the models and experiments to the 100 to 10,000 year prediction. He also addressed the question of whether one could justify testing in materials other than Yucca Mountain rock and obtain results in a relatively short time (e.g., I year) which were relevant to the long time in Yucca Mountain.

Our progress report of December 7, 1988 described the concept of reproducing relevant processes in materials of larger permeability to accelerate the time scale and yet include the effects of concern (e.g., the precipitation of dissolved solids in the rock pore space near the canister). A companion report described some measurement concepts, given that one co:il use a higher permeabllity material.

\section{RESULTS}

The level of effort of our contract prohibits our resolving the issues we define with more than simple analytical means. But, the hope is that the questions and concepts evolved will be useful to the scientific problem solution.

\section{Steam Evolucion in Calculations and Experiments}

We defined an energy ratio which is important to the formation of precipicates near the canister hole. The ratio is the energy carried by steam loss from the hole divided by the energy transported by conduction in the rock away from the hole. The assumption was that if the ratio were large a great deal of water was evolving from the hole as steam, and dissolved solids would be left behind during the vapor evolution which could affect the medium properties and the caniscer enviroment. 
Furthermore, a great deal of steam evolution could cause subsequent formation of large volumes of condensate in such locations as the cunnel or cooler regions of the borehole. The subsequent condensate flow could change the concept of the local flow field for the canister enviroment and for performance assessment.

Since the description of tie energy ratio as a figure of merit, we have read numerous reports of heat pipe effects, repository scale calculations of convection and explicit fracture and effective continuum calculations of flow near a single canister. Some of the early calculations did not allow steam to escape from the borehole. Those calculations forced all convection and conduction to diverge away from the borehole. Wacer transport cowards the hole was ailowed via capillary suction toward the hot dry region in most calculations. The TOUGH code is often used for the calculational analysis.

Tom Buscheck has provided to us the temperature and saturation plots of three different calculations of the same sttuation. One calculation assumed matrix rock alone, one used an explicit fracture geometry and the other used an equivalent continuum model developed by John Nitao. The interesting fact was that with all three models the temperature distribution at 120 days was nearly identical near the borehole and far from the borehole (see Figures $l(a)$ and $l(b)$. Only the equivalent continuum showed the flat spot in the temperature profile characteristic of steam convection energy transport greater than conduction transport.

The associated saturation curves are much more different (Figures 2(a) and $2(b))$. It is probably important that the equivalent continuum result reaches nearly 1008 saturation near $1.7 \mathrm{~m}$. At that saturation, water is most mobile, and steam is not, and probably accounts for the short section of steam convection frow about 1.3 to $1.7 \mathrm{~m}$ (Figure $l(b)$ ).

As an estimate of the energy carried out the borehole as steam, we tried to calculate the net loss of water from the pore space for the "matrix only" case. For reasons that were hard to explain, the water lost near the borehole was less than the water gained in the outer region above 
*gt40rfa/b/e*2-D Radl,1 rdcl;46×1 msh;infiniteheater;swi=.65

TEMPERATURE (DEG. C.) at $\mathbf{1 2 0}$ days

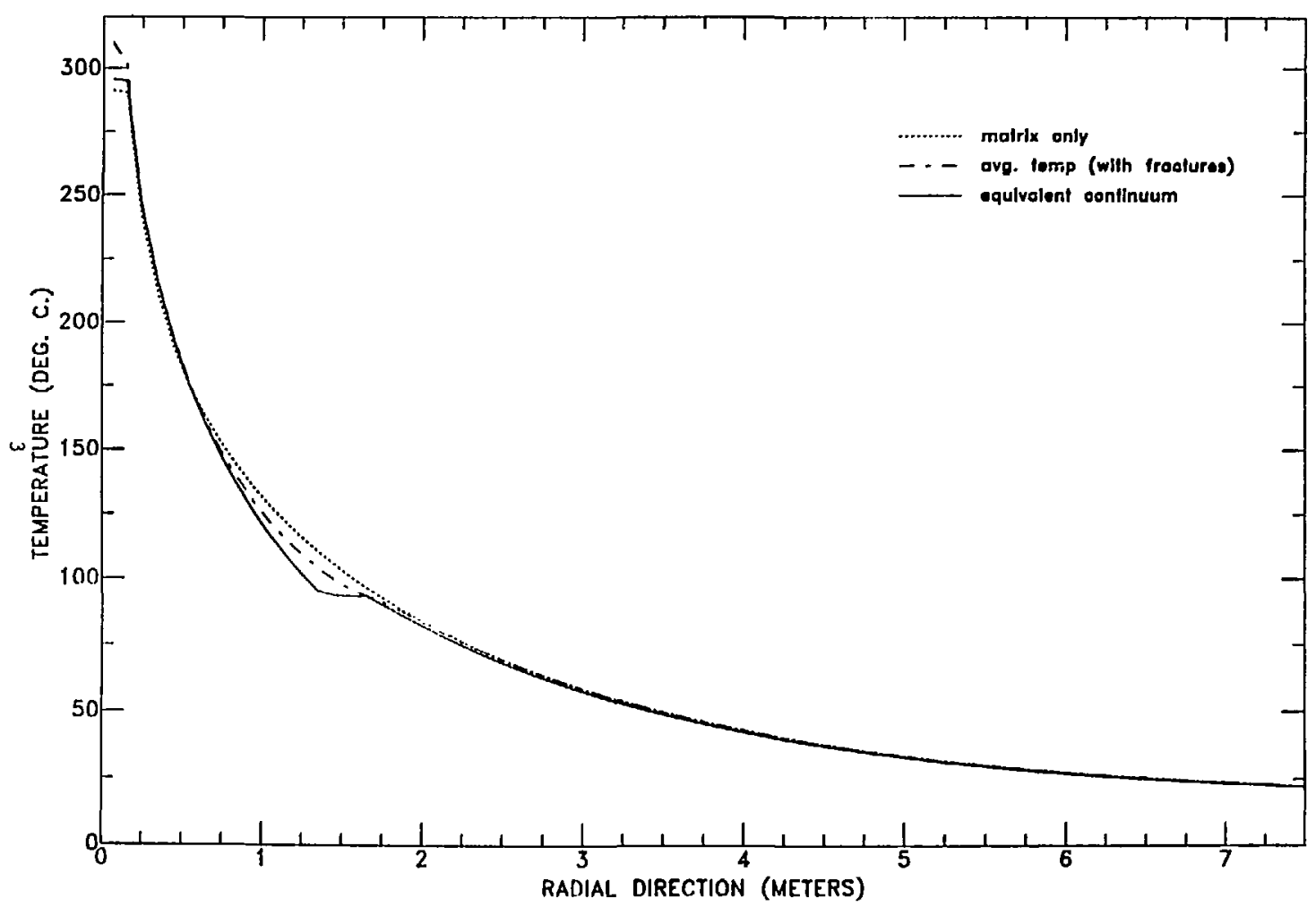

Flgure $1(a)$. Temperature distribution 0-7.5 m from borehole centerline. 


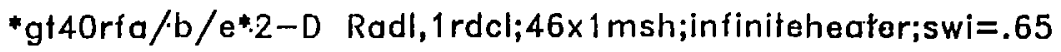

TEMPERATURE (DEG. C.) at 120 days

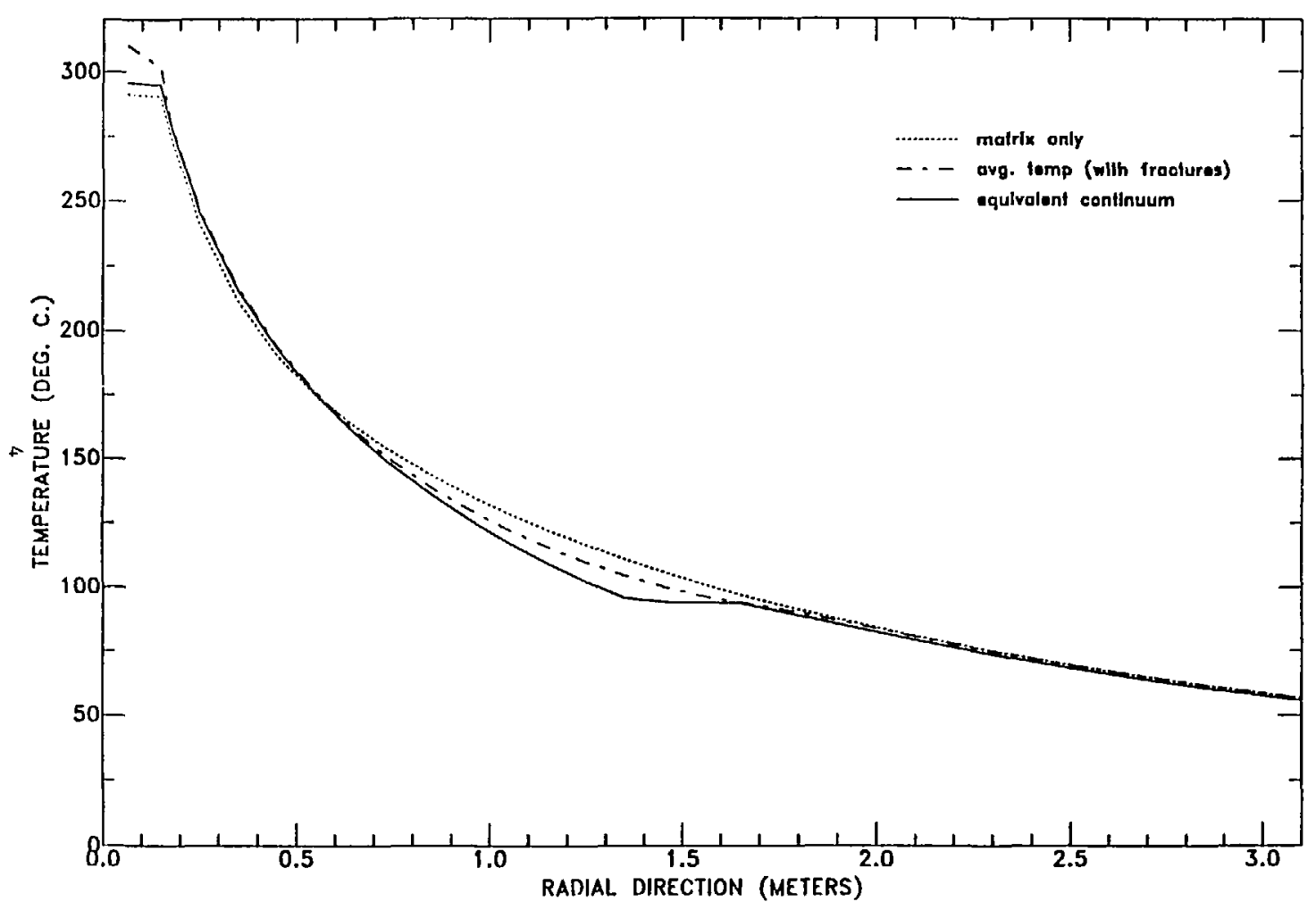

Figure $1(b)$. Temperature distribution $0.3 \mathrm{~m}$ from borehale centerline. 
*gt40rfa/b/a*2-D Radl;rdcl;46×1 msh;infiniteteater;swi=.65

LIQUID SATURATION at 120 days

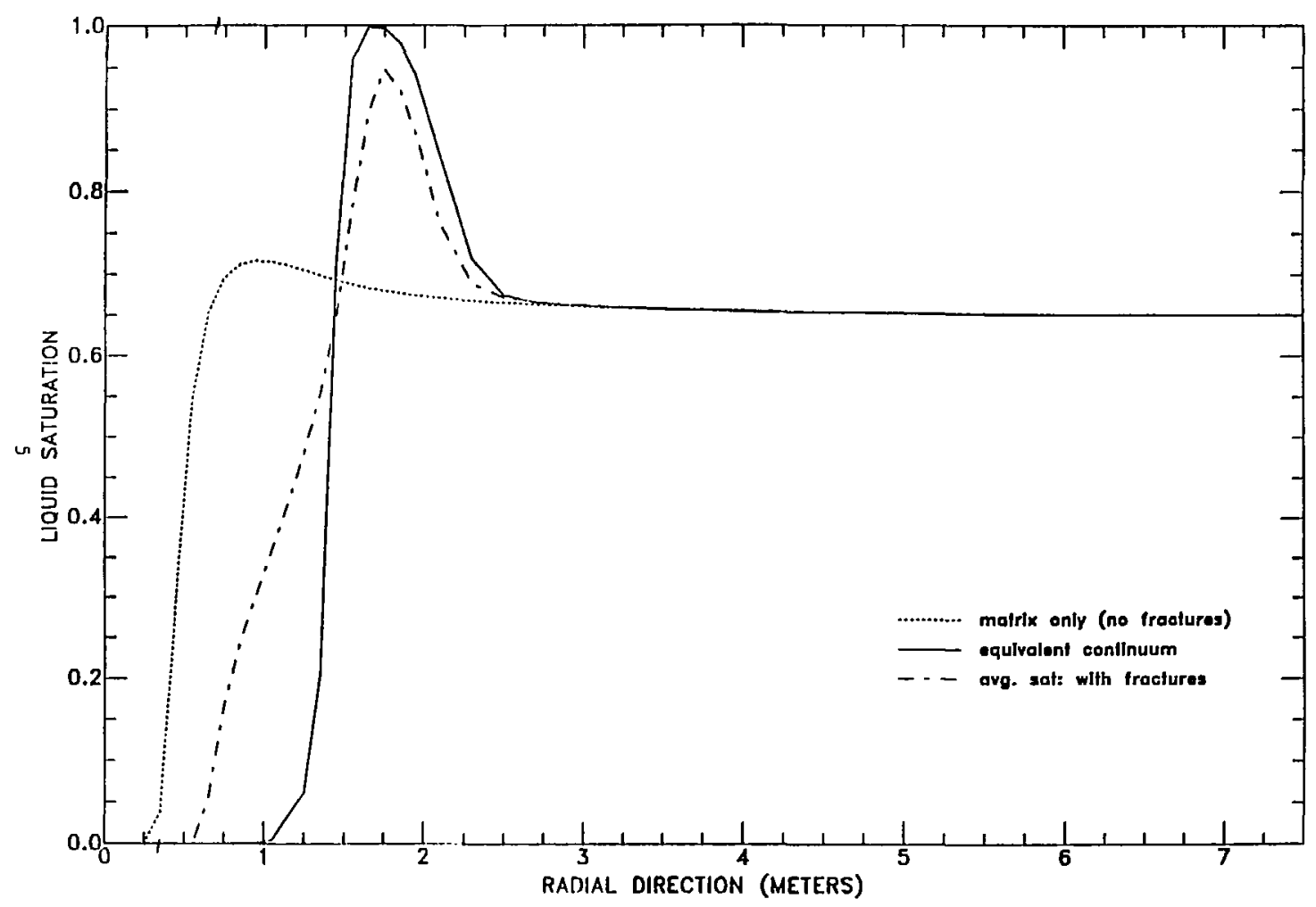

Figure 2(a). Saturation distribution $0.7 .5 \mathrm{~m}$ fram borehole centerline. 
${ }^{*} \mathrm{~g} \nmid 40 \mathrm{rfa} / \mathrm{b} / \mathrm{e}^{*} 2-\mathrm{D}$ Radl;rdcl;46×1 msh;infiniteheater;swi=.65

LIQUID SATURATION of 120 days

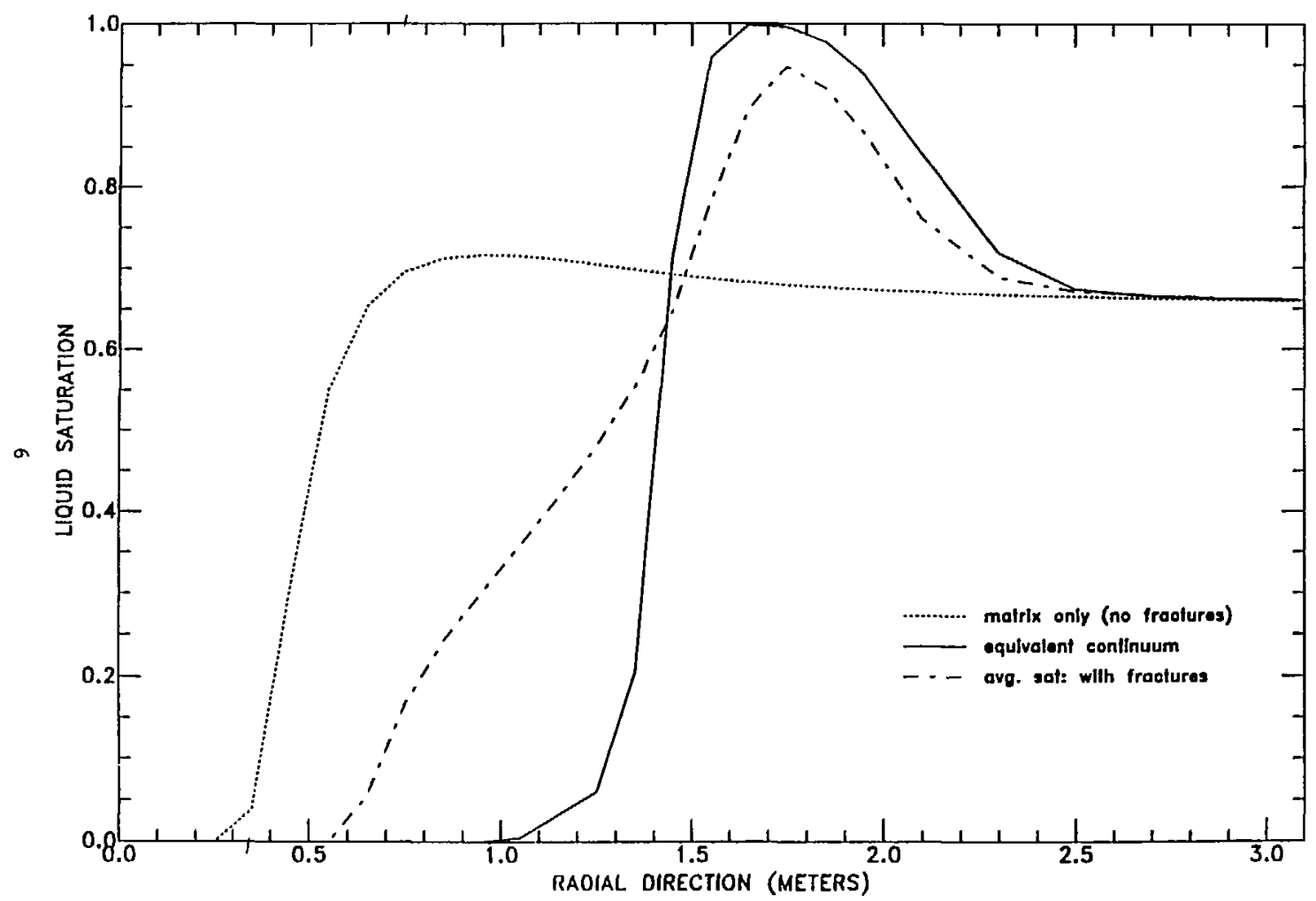

Figure 2(b). Saluation distribution 0-3 m from borehole centerline. 
the ambient saturation. The excess water seemed to be about $50 \%$ of that lost from the dried region. It seemed as though steam flowed into the rock from the borehole or water was drawn up the saturation gradient from afar.

Tou Buscheck pointed out that the model includes a reduction of water density at elevated temperatures and, Indeed, that accounts for the apparent excess water. In fact, the recalculation with the water density correction suggests that the water which evaporated had left the problem. A quick estirate of the heat required to raise water to $300^{\circ} \mathrm{C}$ is 700 calories per gram. The loss of $5 \times 10^{4}$ grams of water over 120 days carrying 700 calories per gram leads to a loss of only 0.08 of the energy input ( $1 \mathrm{Kw} / \mathrm{m}$ ). This compared favorably with the energy calculated to have been lost by flow from the hole. This implies that very little steam flows away from the hole to form condensate in the medium for the matrix only calculation.

The fact of little calculated heat loss in steam from the hole, and the fact that the temperature distribution shows so little perturbation from a normal conduction curve, suggests that conduction strongly dominates the transport of the heater energy. The fact that the dried region continues to grow throughout the calculation also suggests that what precipitation of dissolved solids does occur, due to steam loss from the borehole, is distributed over a relatively large volume of rock and therafore has a small effect on the pore size, water chemistry and permeloilicy.

However, there is still the question about enhanced solution of the rock in the heated region. If the hot water dissolves the local rock and then the water is evaporated, it could change the local pore structure. If so, the rate at which water can flow back into the cooling canister region could be affected.

The more interesting possibility is that the matrix lock could be dissolved in the hot, high pressure region of Figure 3 (from a November 1988 report no. UCID-21571 by T. Buscheck and J. Nitao). Flow toward the 


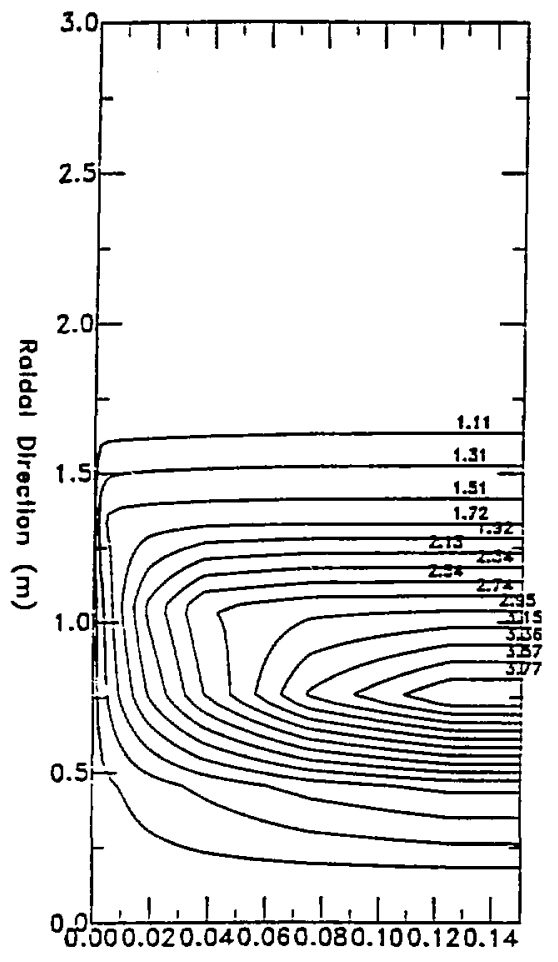

Axial Direction (m)

Figure 3. Contour plot of gas-phase pressure (atm) within the fracture and matrix at the end of the full-power heating stage ( $t=6$ months). Note that axial distances are exaggented by a factor of 10 . 
lower pressure region of the fracture could allow the hot water to flash to steam in or near the crack, dropping the dissolved solids at that point. The possibility of crack healing/clogging may be relatable to the experimental results of Wunan Lin and Bill Dailey.

The results of Buscheck and Nitao do not show extensive capillary transport of water into the dried region near the borehole. This would imply a very low energy ratio and a difficulty of cesting in materials of larger permeabilicy with the same processes dominant. Results of calculations by Christine Doughty and Karsten Pruess (Int. J. Heat Mass 3 . Transfer, Vol 32, No. 1, pp 79-90, 1988) also show reduced heat pipe like flow for relatively impermeable rock. (Note: their calculations do not allow vapor to vent from the borehole). Only the case in which Pruess allows large water flow in fractures toward the hot source does the possibility exist for large deposition of dissolved solids. The reality of the model should be tested because of its significant effect on precipitation of minerals near the heated borehole.

In general, the capillary suction driven flow in the calculations is quice small, because the model used for relative permeability assigns a very low value to water flow at less than full saturation. The Mualem model was developed for soils. I have read of doubts about its validity for extremely low permeability rock. What is known about its validity? Later in this report I'll describe some ideas far better relative permeability measurements.

From comments by Abe Ramirez about the lack of a high sacurated region around the $G$ tunnel heater bole, we would infer (1) that the capillary forces modelled are not high enough to smooth the high saturation peaks calcuiated for two of the three models, or (2) the vapor flow calculaced with the fractured rock model is too great and exaggerates the condensation, or (3) the relative permeability used in the model is too small and doesn't allow the saturation peak to diffuse under reasonable capillary forces. Or, (4) the "matrix only" calculation is the more relevant calculation and fracture flow does not have much effect on the saturation curve (see Figure 2). 


\section{Fracture Mode1s}

The fracture flow modelled by Nitao and Buscheck (Figure 3) is very instructive within the limits of the models. Unfortunately, the assumptions which allow the asymptotic solutions are reasonable if the fractures are of less concern (e.g., small heads, no large pressure gradients in the fracture, fracture pressure much less than capillary pressures). However, any rellef from the assumption of matrix and fracture equilibrium used for the equivalent continuum model is useful for assessment of the possibilicy of faster flow in fractures.

The experiments possible in the time available will almost certainly require that the source pressure be large. Otherwise, flows will be so slow as to be difficult to measure. This means that the models which do not treat a high pressure source condition cannot be checked agalnst the experiments available. There are also those who fear that the climate at Yucca Mountain may evolve to a tropical rain forest, or at least a very large thundershower is likely, that could exceed current estimates of a few millimeters of infiltration a year. This is another reason to model well pressurized fracture flow.

Finally, there is the logical argument that the sinall fractures are safe. How large a fracture may be discovered in Yucca Mountain? Upon drilling a sanister hole, should we test it to assure that all fractures are less than some critical value? Modelling of larger fractures seems important, but it violates some simplifying assumptions that are common.

At what heating rate will the surface of the borehole be an evaporation surface without a large dry region in the rock? Will hot canisters force water into the holes ne cooler canisters? Can permeability asymetries allow flow channels to dominate the flow? If ancient water is trapped in the lithophysae of the rock, can it lead to steam pressures high enough to rupture the rock and allow rock pieces to fall into the hole against the canisters? 
The creep rate of the rock shown in Figure 4 increases by four orders of magnitude per each $200^{\circ} \mathrm{C}$ temperature increase. Will this affect the hole stability and the pore geometry significantly?

Will measurements made in 1 year detect significant effects? It seems that hese questions must be answered if not already addressed by others.

\section{Possible Measurements of Relative Permeability}

Many aspects of the results of Buscheck and Nitac depend upon the value of the relative permeability used for the calculations. The rate of water flow into the heated region, flow into the matrix from a fracture and vice versa, the relief of steam pressure developed in the rock and the possibility of steam rupture of the rock are all processes corrolled by the relative permeability. Hereafter, at the risk of repeating what is well known, are presented some thoughes and calculations on the importance of measurement of representative relative permeability values.

The Darcy flow velocity for liquid transport due to capillary suction $\begin{array}{r}\text { pressure } P_{c}{ }^{i s} \dot{K}_{e f f} \partial P_{c} \\ Q / A\end{array}=K_{m} K_{r} \frac{\partial P_{c}}{\partial S} \frac{\partial S}{\partial x}$

where the term $\mathrm{K}_{\mathrm{m}} \mathrm{K}_{\mathrm{r}}$ aP/aS has been called the fluid mobllity. Usually the capillary pressure as a function of saturation is measured as $P_{c}(S)$. But $K_{r}$, the relat:-'a permeability is not known. The estimate of $K_{r}$ developed by Muale.s uses the coefficients of $P_{c}(S)$, when it is fit by the functional form of van Genuchten.

van Genuchten's form of $P_{c}$ is

$$
\begin{aligned}
& \mathrm{S}_{\mathrm{e}}=\left[1+\left(\alpha \mathrm{P}_{\mathrm{L}}{ }^{\beta}\right]^{-\lambda}\right. \\
& \alpha \text { and } \beta \text { are measured coefficients }
\end{aligned}
$$

$\lambda=1 \cdot 1, \beta, \mathrm{s}_{\mathrm{e}}$ is the effective saturation 


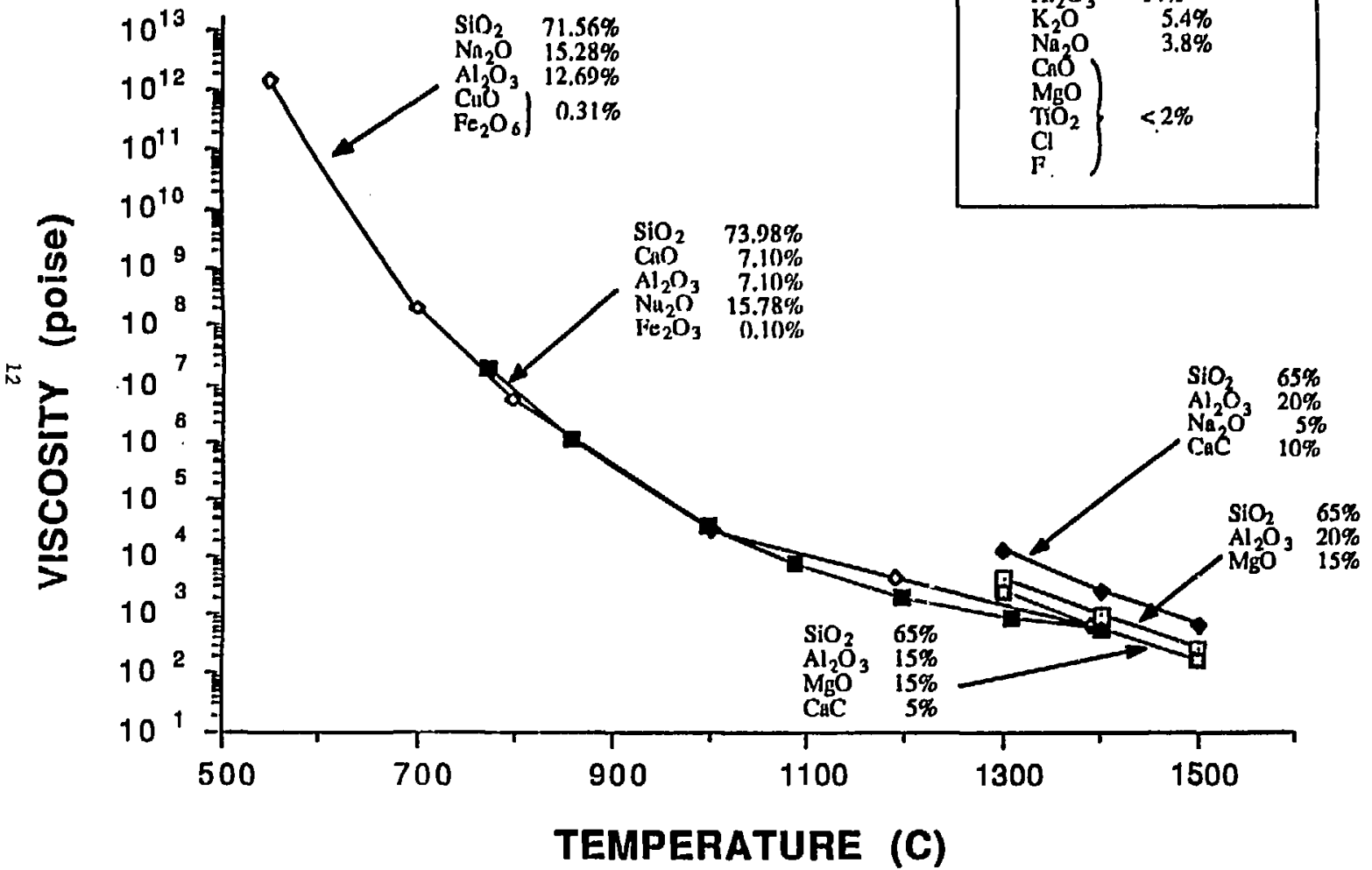

Figure 4. Bock viscosity vs. temperature. 
$s_{e}-\left(S-s_{r}\right) /\left(S_{s}-s_{r}\right)$ with the usual definltions.

Mualem's form for the medium permeabllity is:

$$
K(h)=\frac{K_{\mathfrak{m}}\left[1-(a h)^{\beta-1} \cdot\left(1+|\alpha h|^{\beta}\right)^{-\lambda}\right]^{2}}{\left(1+|\alpha h|^{\beta}\right)^{\lambda / 2}}
$$

where $h-P_{c}$, In our case, and $K_{m}$ is the saturazed medium permeability.

We rewrote $P_{c}$ as a function of $S_{e}$, wrote $K$ as a function of $S_{e}$ and derived the expression for $\mathrm{K}(\mathrm{S}) \partial \mathrm{P}_{\mathrm{c}} / \partial \mathrm{S}$, the water mobility.

It was a very "messy" expression to reproduce here. We developed it as a check of the energy ratio described in our previous report. Using the properties of a zeolitzed tuff from Yucca Mountain, identified as IV-A-Z G4-10, for which $S_{r}=.01, \alpha-.022$ and $\beta-1.236$, and Tom Buscheck's saturation curve of Figure 2 for "matrix only", the capillary flow velocities into the hot dry region are on the order of $K_{m} \cdot 4000 \mathrm{~cm} / \mathrm{sec}$, For $K_{\mathrm{m}}=10$ miero Darcy $=10^{-13} \mathrm{~cm}^{2}$, it is clear that $\mathrm{v}=4 \times 10^{-10} \mathrm{~cm} / \mathrm{sec}$ $-10^{-3} \mathrm{~cm} /$ mo does not provide much of a heat pipe effect. Hence the energy that can be carried out of the borehale as steam can only be with steam that evolves from relatively immobile water.

The relative permeabilty of zeolitized rock at sor saturation is about $10^{-5}$. T. Buscheck's rock for Figures 1 and 2 did not have such a high capillary pressure and hence a somewhat larger relative permeability. The conclusion is still the same. Capillary suction is not wicking water into the hot region at a significant rate in the calculational models. The main reason is the extremely low relative permeability at the lower saturations near the hot region. Are the low relative permeabilities real? How can one determine actual values?

In the expression

$$
\frac{K_{m} K_{r}(S)}{\mu} \frac{\partial P_{c}}{\partial S} \frac{\partial S}{\partial x}-v
$$


the rate of water flow depends upon the saturation. In an imbibition experiment, the saturation fronts may look like Figure 5. Curve A shows a tongue at low saturation, a "wall of water" at about 758 saturation and a slower/shorter wetting range to $100 \%$. Since $\partial S / \partial x$ is small for the congue, and $K_{r}$ is low according to Mualum's model, $\partial \mathrm{P}_{\mathrm{c}} / \partial \mathrm{S}$ must be very large, as is oftun measured.

For curve $B$, there is a distinct sacuration front. Even with $a S / a x$ large, $a P_{c} / \partial S$ cannot compete with a low value of $k_{r}$ to allow wetting ahead of the front at low saturations.

Since $P_{c}(S)$ is measuraile and $S(x)$ is measurable, cannot $K_{r}$ (S) be deduced? Measuring the rate at which a saturation value $s_{1}$ propagates in the material and keeping track of the slope $\partial S / \partial x$ at $s_{1}$ and knowing $K_{m}$. in principle allows an estimate of $\mathrm{k}_{r}\left(s_{1}\right)$. The total flow past a saturation value location is the sum of the saturation increase (total water mass) in front of the saturation value.

Experimental geometries may be chosen to control the shape of the imbibition saturation curve to aid measurement of both gas and liquid relative permeabilities. We realize that $K_{r}$ (air) $=1-K_{r}$ (water) is a convenient form, but it may not be reasonable at intermediate saturations.

It is quite clear that experimental results like those from LLNL $G$ tunnel tests are essential to the guidance of the modelling as well as a test of the modelling.

\section{Long Time Scale Effects}

There are several processes that are not well modelled nor easily measured which can be important over long time periods. Precipitation of dissolved solids was discussed, but there are potential natural analogs. However, the concept of a subterranean pure heat source is unique. Most heat sources in the 


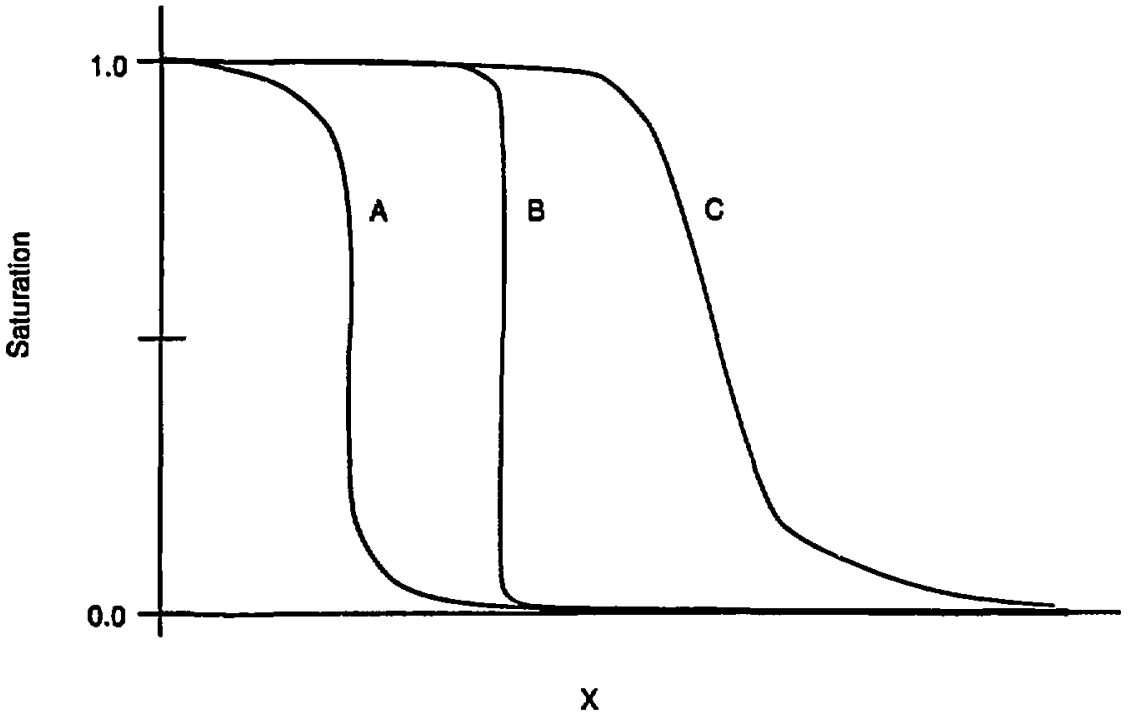

Figure 5. Hypothetical saturation curves during imbibition. 
earth's crust are associated with water or rock flow. Creep is another common process with many examples of creep from smoothly folded strata to the flattened lithophysae in welded tuff. The rock flowed in an apparently plastic, viscous manner. There is common experience with rock spall under thermal expansion or pore pressure increase, but the closure of a $10 \mathrm{~cm}$ gap in 1000 years requires a creep rate of only $2.7 \times 10^{-5}$ $\mathrm{cm} /$ day. Have creep measurements of welded tuff been made at the expected elevated temperatures? We raised this same question in the review of an LLNL borehole stability study plan.

\section{Conclusion}

It is easy to ask questions about processes and it is easy to criticize the treatment of processes that are difficult to model or evaluate. Hopefully, this summary will help to focus attention on important assumptions that are inherent in the current models. ultimately, experiments will test the adequacy of the models, but some processes such as precipitation, creep, thermal mechanical or steam spall and mineral decomposition are so slow as to require very high resolution short term measurement for processes significant over the long time scale. The wide range of behavior hypothesized for fracture flow and the large significance of that flow suggests an experimental program focused on the process under a wide range of conditions.

Finally, the fractures seen in vertical boreholes are necessarily a very small statistical sample of the fractures existent. A careful examination of the range of fracture geometries mapped in Rainier Mesa at NTS leads one to suspect that the fractures madelled of $100-1000 \mu \mathrm{m}$ are not the fractures seeping water in the tunnels. How one deals with larger fractures may be a repository design problem rather than a modelling problem. 